Jaurnal of Environmental Science, Computer Science and

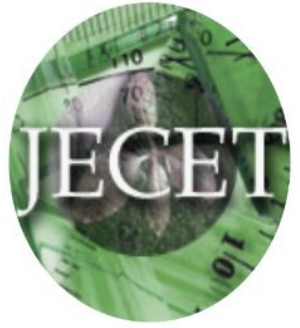
Engineering \& Technology

An International Peer Review E-3 Journal of Sciences and Technology

\author{
Available online at www.jecet.org
}

Section B: Computer Science

Research Article

\title{
Model-View-Controller Framework for Postgraduate Admission and Inter-University Academic Records
}

\author{
${ }^{1}$ Balogun A. O; ${ }^{2}$ Dr.Oye, N. D. \\ ${ }^{1}$ Department of Information Technology, MAUTECH, Yola- Nigeria \\ ${ }^{2}$ Department of Computer Science MAUTECH- Yola Nigeria
}

Received: 03 May 2018; Revised: 27 May 2018; Accepted: 13 June 2018

\begin{abstract}
Web applications have become the primary source of information and transactions over the internet and this has led to high demand for continuously evolving web applications. University administrators and other stake holders depend on them for many of their operations including postgraduate admissions. Even though many institutions have independently developed systems to handle postgraduate admission processing, there is need for the development of a system that will serve all Nigerian institutions in order to securely and seamlessly integrate all the various aspects involved in the domain. Nowadays there are different forms and technologies for implementing web applications without much concern for their architectural model. Without a good and stable architecture those applications may fail during their operations. This study therefore focuses on an architecture that conforms to current best practices in creating web solutions. The Model-View-Controller (MVC) software engineering architecture has been widely embraced as an approach for developing webbased systems that contain a server-side programming component, particularly for those requiring database access. Of the various possible methodologies for carrying out this study, the researcher made particular choices: Use Case texts, Use Case diagram, Domain class diagram, design class diagram (DCD) and ASP.Net implementation. Database design was done using T-SQL and Local DB feature in Microsoft Visual Studio 2012. Programming language was $\mathrm{C \#}$ and the design was implemented using the Internet Template. The implemented design was tested and the results are shown. This study has been able to provide an object oriented analysis and design (OOAD) for inter-university postgraduate admission and academic records domain using UML
\end{abstract}


artifacts. Suggested further studies include expanding the framework to include other forms of postgraduate admissions and incorporation of interactive multimedia.

Keywords: Academic Records, Model-View-Controller, Web Application, Transcripts, T-SQL and Local DB

\section{BACKGROUND OF THE STUDY}

Web applications have become the primary source of information and transactions over the internet. This has led to high demand for continuously evolving web applications. The web industry foresees frequent shifts in the requirements and architecture of web applications throughout their lifecycle ${ }^{1}$. Nowadays there are different forms and technologies for implementing web applications without much concern for their architectural model. Without a good and stable architecture, those applications may fail during their operations. Thus, experts have focused a lot of attention on exploring different architectural models for creation of web solutions with the aim of determining how best to use them ${ }^{2}$.

The Model View Controller (MVC) software engineering architecture has been widely embraced as an approach for developing Web-based systems that contain a server-side programming component, particularly for those requiring database access. MVC isolates the business logic from the user interface, with the goal of creating applications that are easier to manage and maintain because designers can modify the visual appearance of the applications and programmers can modify the underlying business rules with fewer harmful side effects ${ }^{3}$.

On another dimension, there is a shift in global development agenda and the Nigerian government is introducing some reforms in our educational system with a view to repositioning it in line with the vision of Information and Communication Technology (ICT) ${ }^{4}$. The need for technological innovation has led to a revolution in the development of technological facilities that are applicable to keeping and management of school records. Making use of ICT for record keeping and management will go a long way in raising the standard of school records anywhere in the world. Educators are fast realising the need for using computer for the keeping and management of school records. This would be convenient to both administrators and academic and non-academic staff in tertiary institutions ${ }^{5}$.

For certain reasons, students who are yet to conclude their programmes of study sometimes apply for transfer to continue their studies in other institutions. Nigerian Universities accommodate this in their administrative operations; for instance, the University of Nigeria, Nsukka in its 2015/2016 guidelines ${ }^{6}$ for inter-university transfer to UNN, stipulates that "transfer students shall possess the minimum University of Nigeria and Departmental entry requirement prior to entry into university from where they are seeking transfer. Transfer students shall make a minimum cumulative grade point average of 3.00 on a 5.00-point scale to qualify for consideration and should not have spent more than two (2) academic sessions in his or her former university ${ }^{6}$. Thus, universities, when processing transfer cases like the aforementioned, would require some sort of verification mechanisms to ascertain the stipulated transfer requirements and such systems should be expected to be as efficient and error-free as possible.

On the other hand, admitting universities customarily require official copies of students' academic records as one of the prerequisites for processing admission applications from graduates coming in from other institutions into their postgraduate programmes. Taking the case of the University of Ibadan for example, the 2015/2016 Admissions Guide 7 into the postgraduate school states that "official transcripts of all degrees completed are to be mailed to the Postgraduate School. To prevent delays, applicants should make adequate arrangements with their Registrars to provide transcripts before the stipulated 
deadline. When an applicant fails to provide required documents, (transcripts, etc) his or her admission chances suffer in relation to those whose credentials are complete ${ }^{7}$.

Other corporate bodies or individuals also make requests for transcripts when considering candidates who apply for scholarship or for other purposes. This is to verify and ascertain a candidate's or applicant's eligibility or otherwise for the purpose at hand. As it is with disparate systems, the issuance and receipt of student academic records across different Nigerian university systems has been fraught with a number of problems affecting the candidate who is applying for transcript, the alma mater which is to issue and send the transcript, and the admitting institution that is expected to receive and consider the transcript. Such problems include postal and courier services delay, proneness to being tampered with/falsification or even the possibility of outright loss.

Letters of recommendation or Referee's Reports are given very serious consideration in postgraduate school admission process ${ }^{7}$. There is the need to simplify the processes and shorten the time normally involved when applicants need to get these forms completed by their referees of choice who usually would prefer easier and quicker ways of carrying out the process. When applications are made for postgraduate studies, there is the need to have some means by which transcripts and letters of reference can be processed seamlessly between the different Nigerian Universities that are involved and the referees that have been chosen to make such letters of reference for the candidates applying for admission. Indeed, some universities have developed electronic/online systems for receiving letters of reference while some others also have in addition some web-based provisions for their alumni to be able to remotely pay and apply for transcripts.

The prevalent situation however is such that a university that may be willing to electronically send transcripts may not be matched by another that is enabled to electronically receive while those willing to electronically receive may be dealing with those not able to electronically send. By and large, the practice among Nigerian universities still largely utilize the surface mail systems in sending and receiving transcripts and in processing forms/letters of reference.

\section{STATEMENT OF THE PROBLEM}

The key problem focus of this research is to provide an object oriented analysis-based design for the proposed system using UML artefacts. This is because present development in web technologies has simplified development of web applications with little or no consideration to the question of design ${ }^{8}$. Current development environments have made it very easy to produce web applications that they have the unfortunate side effect of encouraging developers to develop and evolve web applications in the absence of serious or rigorous analysis and design. Solving a problem of this nature and size can be very tasking and quite difficult to completely achieve in one fell swoop. However, going beyond mere presentation of models, there is a need to identify the functional requirements for a system suitable for the problem domain and hence develop a framework that will provide basic tools and behaviour for the system which software developers in this domain may use to build specific solutions without having to go through all the cumbersome ground work already provided in a framework.

Away from the academic, the study aims at solving some problems currently experienced by stake holders in the area of university postgraduate applications and admissions. The manual system of issuing and receiving transcripts of students' academic records, like many other human-run systems, is susceptible to various possibilities of corrupt practice. In a domain as important as postgraduate admission, it should be expected that universities in the near future begin to consider how to make the ntire process of issuing and receiving of transcripts strictly a business between the alma mater and the 
admitting institution.Another issue is that of the risks and avoidable costs on the side of the applicants who travel back to their alma maters for the purpose of applying for transcripts. On the side of the alma maters and admitting institutions, manual sorting of payment records, matching them with corresponding requests for transcripts and matching referee forms with the right applicant's application form is quite tedious, time consuming and error-prone. A system that that automatically matches an applicants' form with their supporting documents would be better.

Referees are customarily required to make letters of reference as part of the process in gaining admission for postgraduate studies. When the system does not provide an easy way for such referees to do so, applicants do experience some reluctance on the part of their chosen referees due to the stress of manually filling the forms and possibly sending the completed forms by post. Presently, most Nigerian universities only independently operate disparate portals for application into their schools of postgraduate studies. The idea behind this research work is to come up with a design of an application that can be used by all Nigerian universities for the purpose of postgraduate admissions.

\section{AIM AND OBJECTIVES OF THE STUDY}

The aim of this study is to provide an MVC framework that can be used by developers to develop web applications that will handle application for postgraduate studies admission with incorporated functionalities to process student transcripts of academic record and referee forms among universities.

The objectives are as follows:

i. Define use cases for the system

ii. Present a domain model for the system

iii. Draw interaction diagrams (particularly sequence diagrams) for use case realization of system events to obtain the models, controllers and system operations

iv. Collate Design Class Diagrams (DCD) required for coding/implementation

v. Implement the resulting design using ASP.Net MVC (Visual Studio IDE)

Significance of the Study: Academically, this work provides a ground work or foundation upon which developers who wish to develop this type of application can base their work specifically using the MVC architecture. This is so because this framework provides the core necessities required to achieve the development of a functional system in a domain such as this with little additional embellishments. Again, this research work may serve as the beginning of further work to result in a better fine-tuned framework to achieve more robust systems. Having laid some germane skeletal maps for the system, it would be easier for other experts in the field to extend the depth and strength of the framework.

This work signifies a simple achievement of a fairly robust framework for a very complex system. The integration of a relatively simple database system with an ORM framework such as Entity framework greatly pulls off the hood hanging over the face of such a system as this. On the other hand, the result of this research work, if adopted and implemented, will be significant to graduate students who pursue further studies in other institutions other than their alma maters. It would bring a lot of relief from the stress that has become associated with postgraduate admission applications. Universities will also benefit from this work as it would offer a largely automated and less stressful alternative in the process of graduate admissions processing. Even for institutions that already have some sort of online systems for postgraduate application, an inter-university system such as this portends greater advantage in many ways. This study will also be very significant to fraudsters who over time have made a living out of transcript and result forging as their business will be put to a serious threat. This will be so because the entirety of the process of issuance and receiving of transcripts would be completely taken out of their 
realm of operation. The study will also be significant to those who previously may have declined to make references for applicants because they were avoiding the hassles of completing hard copy referee forms coupled with the stress of surface mailing associated with postal agencies or courier service providers in sending such completed forms.

Scope and Limitations of the Study: The scope of this study covers the design and implementation of an inter-university web application for admission application, transcript service (request, issuance and receipt), making references and application processing. Only the main success scenarios (MSS) were given in-depth consideration in this study. The design provides for the possibility of the system being used by all Nigerian universities. The limitation of the research was mainly the researcher's resources vis-à-vis the scope of the scope of research domain.

\section{LITERATURE REVIEW}

Modeling Web Applications: According to Schwinger and Koch ${ }^{9}$, it is not common to model Web applications in practice which they consider to be unfortunate. They argue that a model based approach provides a better alternative to the ad-hoc development of Web applications and its inherent problems including insufficient fulfillment of requirements, faulty specification, or missing system documentation. Models represent a solid starting point for the implementation of a Web application taking into account static and dynamic aspects of the content, hypertext, and presentation levels of a Web application.

While the content model of a Web application which aims at capturing underlying information and application logic is similar to the corresponding model of a non-Web application, they see a need to consider the hypertext which is particular to Web applications. The hypertext model represents all kinds of navigation possibilities based on the content. The presentation model maps hypertext structures to pages and their links thus represent the graphical user interface. The inclusion of context information, such as user, time, location, and device used, and the adaptation of the Web application which is "derived" from this information, has gained increasing attention in modeling efforts. This is undoubtedly a consequence of ubiquitous Web applications that have become increasingly popular. Engineering disciplines have successfully used models to reduce complexity, document design decisions, and facilitate communication within project teams. Modeling is aimed at providing a specification of a system to be built in a degree of detail sufficient for that system's implementation. The result of a modeling process is models representing the relevant aspects of the system in a simplified and - ideally - comprehensible manner. Computer science has also been using the modeling approach to develop software for some time. In this field, the object of modeling is the application to be created. Figure 1 shows that the scope of modeling spans along three orthogonal dimensions.

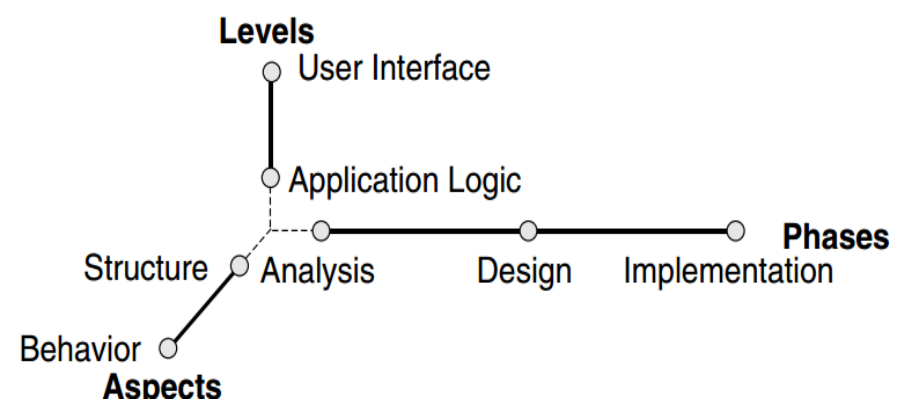

Figure 1: Requirements of Software Engineering (Schwinger \& Koch, 2006. Pg 40) 
The first dimension traditionally comprises the application logic level and the user interface level in the sense of an encapsulation of the "what" and "how" of an application. Aspects known as structure (i.e., objects, their attributes, and their relationships to other objects) and behavior (i.e., functions and processes), both of the application logic and the user interface, form another dimension. Since an application cannot be developed "in one shot", but has to be gradually refined and expanded during the development process, the development phases form the third application modeling dimension. Through successive refinements the requirements identified in the requirements analysis are transformed to analysis models first and design models later, on which the implementation will be based.

Applying UML and Patterns: According to Larman ${ }^{10}$, learning the core skills in object-oriented analysis and design (OOA/D) are essential for the creation of well-designed, robust, and maintainable software using object technologies and languages such as Java, C++, Smalltalk, and C\#. Knowing an object-oriented language (such as Java) is a necessary but insufficient first step to create object systems. Knowing how to "think in objects" is also critical. He advocated an approach to OOA/D using the Unified Modeling Language (UML), patterns, and the Unified Process. He placed emphasis on the mastery of the fundamentals, such as how to assign responsibilities to objects, frequently used UML notation, and common design patterns.

Beyond UML as a standard diagramming notation, he says there are more critical object-oriented things to learn; specifically, how to think in objects-how to design object-oriented systems. The UML according to him is not OOA/D or a method, it is simply notation. He stated that it is not so helpful to learn syntactically correct UML diagramming and perhaps a UML CASE tool, but then not be able to create an excellent design, or evaluate and improve an existing one. This is the harder and more valuable skill. Nevertheless, one needs a language for OOA/D and "software blueprints," both as a tool of thought and as a form of communication with others. Therefore, he explored how to apply the UML in the service of doing OOA/D, and covered frequently used UML notation. But the emphasis is on helping people learn the art and science of building object systems, rather than notation.

According to him, the critical questions in the design of a system are how should responsibilities be allocated to classes of objects? How should objects interact? What classes should do what? He therefore surmised that certain tried-and-true solutions to design problems can be (and have been) expressed as best-practice principles, heuristics, or patterns - named problem-solution formulas that codify exemplary design principles. His book, hence teaches how to apply patterns to support quicker learning and skillful use of these fundamental object design idioms.

Analysis and design: Object oriented analysis and design (OOA/D) and all software design is strongly related to the prerequisite activity of requirements analysis, which includes writing use cases. Analysis emphasizes an investigation of the problem and requirements, rather than a solution. Figure 2 depicts the various development skills. For example, if a new computerized library information system is desired, how will it be used? Analysis is a broad term, best qualified, as in requirements analysis (an investigation of the requirements) or object analysis (an investigation of the domain objects). Design emphasizes a conceptual solution that fulfills the requirements, rather than its implementation. For example, a description of a database schema and software objects. Ultimately, designs can be implemented. As with analysis, the term is best qualified, as in object design or database design. Analysis and design have been summarized in the phase do the right thing (analysis), and do the things right (design). 


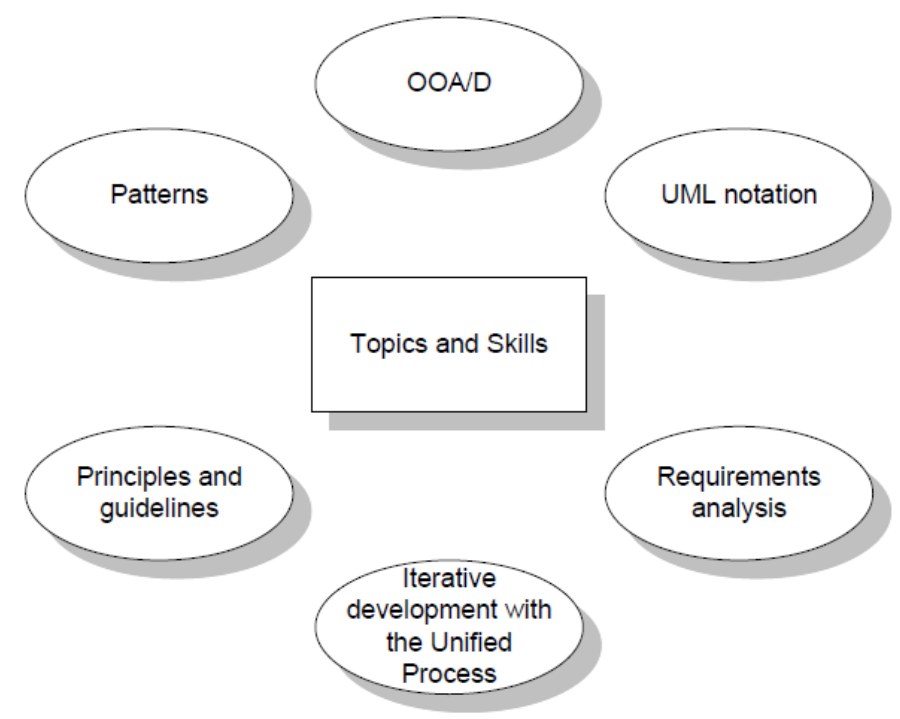

Figure 2: Software Development Skills ${ }^{10}$

Model example of OOAD: This example gives a birds-eye view of a few key steps and diagrams, using a simple example - a dice game in which a player rolls two die. If the total is seven, they win; otherwise, they lose.

Define Use Cases

As shown in Figure 3 and 4, requirements analysis may include a description of related domain processes; these can be written as use cases.

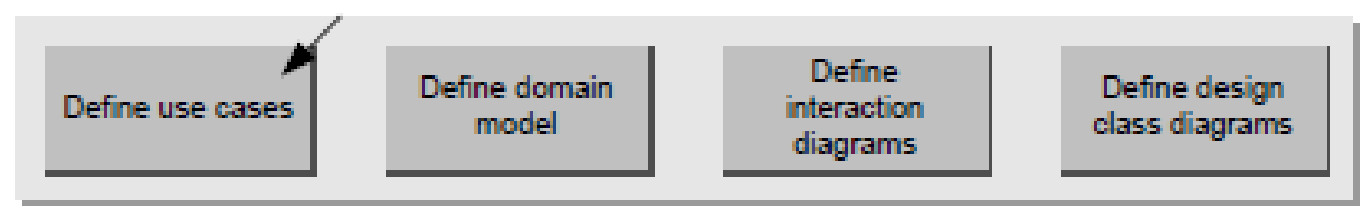

Figure 3: First Step in Requirements Analysis ${ }^{10}$

Use cases are not an object-oriented artifact - they are simply written stories. However, they are a popular tool in requirements analysis and are an important part of the Unified Process. For example, here is a brief version of the Play a Dice Game use case:

Play a Dice Game: A player picks up and rolls the dice. If the dice face value totals seven, they win; otherwise, they lose.

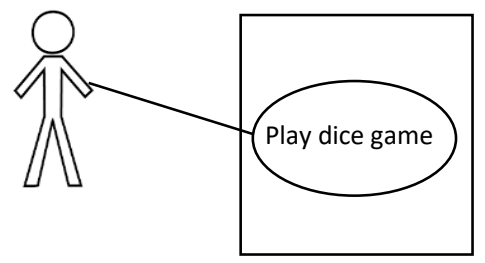

Figure 4: Play a dice game 
Academic Records and Transcripts: In any school setting, examination is a part of the system and record keeping of the results is taken very seriously. In the university system in particular, special attention and even departments or units are sometimes dedicated to examinations and records. It is from these records transcripts can be issued when needed. A transcript is a list of all the courses you have taken during a programme of study, with the result you obtained, and sometimes the credit value of each course. If you have completed your study, the transcript should also show your final award usually as a class or $\mathrm{CGPA}^{11,12}$. Transcripts are sometimes considered to be the same as student academic records but Welch ${ }^{13}$ in her paper makes a distinction between the two: When discussing the recordkeeping function of an institution, a distinction should be made between the terms "academic record" and "transcript." An "academic record" is an internal document or electronic image maintained by the institution's registrar that reflects the unabridged academic history of the student at that institution. It is a chronological listing of the student's total quantitative and qualitative learning experiences and achievements and may include any personal information pertinent to the student.

Information peripheral to the immediate academic experience, such as personal characteristics and experience, family background (including parent name and address), secondary school background, aptitudes, personal interests, entrance examination scores, non-academic awards or honors may also be included in the academic record, but should not be incorporated into the transcript.

A traditional "transcript," on the other hand, is information taken from the student's more extensive academic record at the request of the student or former student. The transcript may be a facsimile of the academic record or a subset of the academic record that minimally, includes the extent and quality of all work attempted at the institution issuing the transcript. A transcript is a legal document which contains the signature of an institution's certifying official, the embossed institutional seal, and the date of issue ${ }^{13}$.

Transcripts are to be made available in a form or format that will be suited to the purpose for which it was requested. Whereas scholarship boards that request for student transcripts may be more interested in the scores obtained by a student or a set of student for the purpose of screening or thinning down the number of applicants, a postgraduate school may not only be interested in the scores or even the course codes but rather in the course titles. This is because postgraduate schools would want to determine from the transcripts the eligibility of the applicant for the particular field of study he/she intends to pursue since generally the courses previously taken by an applicant at say the undergraduate level will be the determinant of his eligibility for a particular area of specialization at postgraduate level.

Welch ${ }^{13}$ goes further to say that whether transcripts are hand copied, photocopied, or computer generated, it is the responsibility of the issuing institution to be certain that the document transmitted as a transcript is appropriate for the use intended, that it contains at least the essential data elements specified, and that it is consistent with the legal guidelines under which the institution operates.

It is the practice for postgraduate schools to demand for an applicant's transcript of academic record as a prerequisite for processing admission. Requests for official transcript of academic records of students and alumni are on the increase. Other than the final statements of results or certificates issued to students and alumni, educational bodies and other institutions are more interested in the detailed academic records of applicants showing attained grades and performance on each courses/subjects taken ${ }^{14}$.

Student Information Management System (SIMS): A student record is any written information about a student which can be described in terms of their contents, use, and storage medium. The maintenance of extensive, accurate, historical, and current data about individual students is essential to the functioning of schools and can promote effective educational practices ${ }^{15}$. Problems exist in the 
management of student records in academic institutions. This has led to the design and implementation of a number of Student Academic Record Management Systems.

Eludire $^{16,}$ states that a number of problems associated with student academic record management include improper course registration, late release of students' results, inaccuracy due to manual and tedious calculation and retrieval difficulties/inefficiency. In most cases the data generated by academic institutions are usually created in non-delineated files for use by different departments/units within the institutions with the same data appearing on several of these files. This means that a simple change of address would have to be processed in two and probably three or four places, depending on the number of other files on which these data appears. The development of database concept is the answer to these problems where the amount of redundant data is reduced and the possibility that data contained on a file might be inaccurate because they were never updated

Clements ${ }^{15}$ touched on two issues that accompany the automation of student records despite the advantages offered by automated systems. These issues are the costs and reliability of the computer systems and secondly the inability of system developers to distinguish between automating a paper system of student records and designing a system that exploits the advantages and utilities offered by computers and ICT tools. A well-conceived and implemented automated student record system can reduce the costs of handling the paperwork associated with record keeping. Even when such a system proves initially expensive and actual reductions in current costs are not achieved, it is justified given future savings and efficiency. Nevertheless, system developers and implementers have to contend with two major concerns in order to maximize the cost-saving benefits of an automated system.

First, it is sometimes thought that automated data systems do not result in actual savings. Granted there are computer purchase costs and personnel needed to maintain the system. These costs, while not minimal, should pay for themselves with the usefulness of the data and the reduced time data providers have to spend on data collections. Concerns about the reliability of computers may also lead some staff members to continue keeping their old records, "just in case." In fact, during the early stages of automating data it is often wise to maintain the data in two places, so that glitches can be resolved before relying solely on the automated system. Once the system is fully functional, however, the back-up paper system is no longer needed.

Another mistake often made is that organizations automate a paper system instead of completely redesigning a system that takes advantage of the computer's capabilities as they exist now as well as thinking about future possibilities. Reconceptualising a system that contains virtually no paper, means that redundant activities such as entering data can be avoided. If, however, you merely automate the paper system, you may achieve fewer savings than could be achieved through a newly-conceived automated system ${ }^{15}$. Information systems used to manage student data have been referred to in various ways: Student Information Systems (SIS), Student Management Information Systems (SMIS), Student Data Systems (SDS), Student Data Warehouse (SDW), Student Academic Information Systems (SAIS), or Student Information Management Systems (SIMS) ${ }^{17}$.

Barrett ${ }^{18}$ encapsulates the essence of a student information system. He defines SMIS as "an integrated software package that maintains, supports, and provides inquiry, analysis, and communication tools that organize student accountability data into information to support the educational process". A result management system (RMS) is only an aspect of a complete SIS package.

Student Information Management System (SIMS) provides a simple interface for maintenance of student information. It can be used by educational institutes or colleges to maintain the records of 
students easily. The creation and management of accurate, up-to-date information regarding a students' academic career is critically important in the university as well as colleges.

Student information system deals with all kind of student details, academic related reports, college details, course details, curriculum, batch details, placement details and other resource related details too. It tracks all the details of a student from the day one to the end of the course which can be used for all reporting purpose, tracking of attendance, progress in the course, completed semesters, years, coming semester year curriculum details, exam details, project or any other assignment details, final exam result and all these will be available through a secure, online interface embedded in the college's website. It will also have faculty details, batch execution details, students' details in all aspects, the various academic notifications to the staff and students updated by the college administration. It also facilitate us explore all the activities happening in the college, Different reports and Queries can be generated based on vast options related to students, batch, course, faculty, exams, semesters, certification and even for the entire college ${ }^{19}$.

Postgraduate Application: The process of applying for postgraduate studies involves a number of things which customarily includes the completion of an application form by the applicant. The Guide to Completing the Online Application Form 2015/16 for the University of Cambridge states that "An application consists of the completed application form itself and the submission of all supporting documentation relating to the application"11,12. Although application forms by themselves are insufficient for securing admission without other concomitant papers and documents as stipulated by the admitting institution, they are nevertheless very crucial in the scheme of the admission process. A survey of some postgraduate admission application forms ${ }^{20,21}$ shows that they are instruments by which the admitting institution collects information of the applicants as they relate to bio-data, academic background and areas of interest of study or research including sundry information as may be deemed necessary by the applicant or the admitting institution.

\section{MVC - ASP.Net implementation}

The history of MVC: The term model-view-controller has been in use since the late 1970s and it was conceived as a way to organize some early GUI applications. Some of the fine detail of the original MVC pattern was tied to Smalltalk-specific concepts, such as screens and tools, but the broader concepts are still applicable to applications - and they are especially well suited to Web applications ${ }^{22}$. Interactions with an MVC application follow a natural cycle of user actions and view updates, where the view is assumed to be stateless. This fits nicely with the HTTP requests and responses that underpin a Web application.

The MVC architecture forces a separation of concerns - the domain model and controller logic is decoupled from the user interface. In a Web application, this means that the mess of HTML is kept apart from the rest of the application, which makes maintenance and testing simpler and easier. It was Ruby on Rails that led to renewed mainstream interest in MVC and it remains the poster child for the MVC pattern. Many other MVC frameworks have since emerged and demonstrated the benefits of MVC — including, of course, ASP.NET MVC.

\section{METHODOLOGY}

Use Case: Use cases are not an object-oriented artifact — they are simply written stories! However, they are popular tool in requirements analysis and are an important part of the Unified Process. A use case is not a diagram; it is a text conveying the story of a scenario of events and interaction that occur between an actor/user and a system. For the proposed system, this work put a lot of attention and emphasis on writing, use cases and clearly documenting them. This is considered very important because in 
developing a framework of this nature, the use cases are most likely the starting point from which this work would be understood by other academics who may be interested in this work.

Use cases have been written to cover all the scenarios between the proposed system and all the identified that use the system. It is in the writing (and rewriting) of system use cases that the discovery of various system events that occur between the actors and the system can be made, which in turn constitutes or forms the basis of the design and development of the software system. The use diagram, which simply is a pictorial presentation of the written use cases, is drawn at the end of the writing of the use cases.

Use Case Diagram: Having written use case texts, use case diagrams are drawn. This is important because it shows a picture at a glance with very few words how a system's users interact with it- i.e. the system's requirements. The Use case diagrams were used to represent the actors i.e. entities including people outside the system that interact with the system. It clearly presented the tasks that the system supports and also shows the associations between the two, i.e. the actors and the tasks. Use Case Diagrams were also used because they are commonly used in almost every project because of its helpfulness in exposing requirements analysis and planning the project. During the initial stage of the development, some of the use cases were defined, but as the development continued, more of them became visible and incorporated.

Class Diagram: Class Diagrams are helpful because they are used in nearly all Object Oriented software designs such as this work involves. They have been used in this work to describe the classes of the system and their relationships to each other. Object Oriented Programming exploits much of the features of Class diagrams and this is very important to this project; class name, list of the attributes and variables for each class and the list of methods associated with each class. The methods were named in very suggestive and connotative ways to aid comprehension.

Object Interaction Diagram: Sequence diagrams and collaboration diagrams are two types of interaction diagrams used for object oriented design ${ }^{17}$. Sequence diagrams are more useful when the interest is to have messages in their timely order or manner, but they do clearly show the collaborative dimension of the interacting objects. However, it is less clumsy with notes and message numbers as the case is when using collaboration diagrams. The design of this work was done using Sequence Diagrams because the alternative (collaboration diagrams) for such a relatively large system as this is considered by the researcher as one that may becloud the simplicity of the framework this research aims at providing. As shown in chapter four, the sequence diagrams present quite clear details of how operations within the application are carried out, including what messages are sent and when. These have principally depicted the application in two dimensions: time and participating objects.

\section{DESIGN AND IMPLEMENTATION}

Presentation of the accomplishment of the research aim and objectives constitute the focus of this chapter. Therefore, it is a tripod of the analysis, design and implementation of the proposed system. This chapter aims at clearly defining how the research problem was solved and capturing the design in ways that are easy to communicate, to review and to implement. Programming may be fun, but developing quality software is hard. In between the nice ideas, the requirements, or the "vision," and a working software product, there is much more than programming; that forms the focus of this research in general and accounts for the content of this chapter in particular. The presentation of the material bears a direct mapping with the research objectives as stated in chapter one; definition of use cases, presentation of domain model, drawing of interaction diagrams, defining design class diagrams and finally design implementation. Figure 5 shows the Use Case Diagram for the system. It shows the six 
Use Cases explained above with two of them being included Use Cases. The diagram also shows six actors: System Admin, Guest, Applicant, Referee, Admitting School and Alma Mater. Whereas an Applicant must have played the role of a Guest before playing the role of an Applicant, a Guest does not necessarily have to become an Applicant. For any given school, it can play both roles of Admitting School and Alma Mater but not the two roles at the same time (for a given applicant).

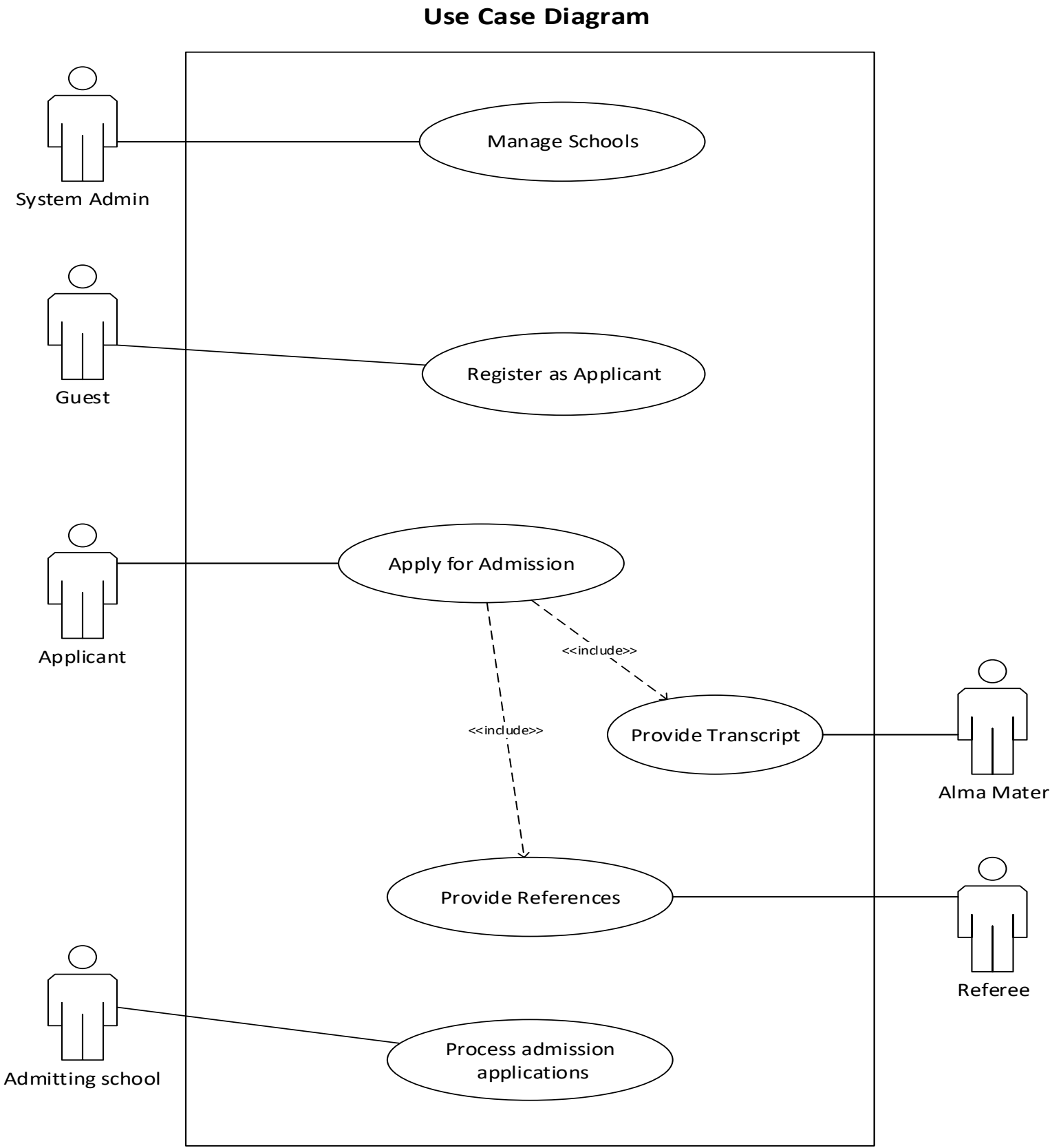

Figure 5: System Use Case Diagram 


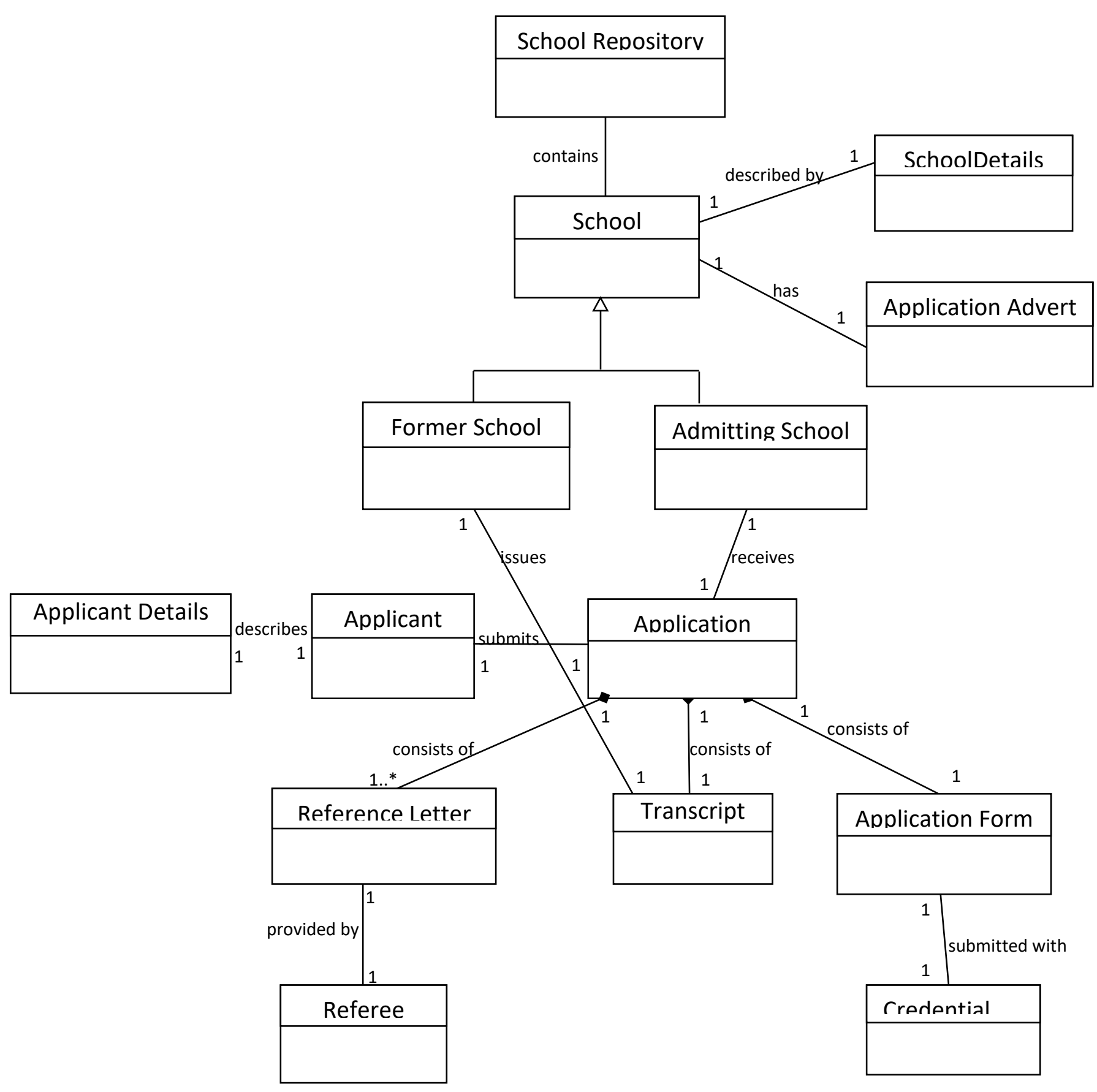

Figure 6: Postgraduate Admission Application Domain Model 


\section{Provide Transcript and Process Applications}

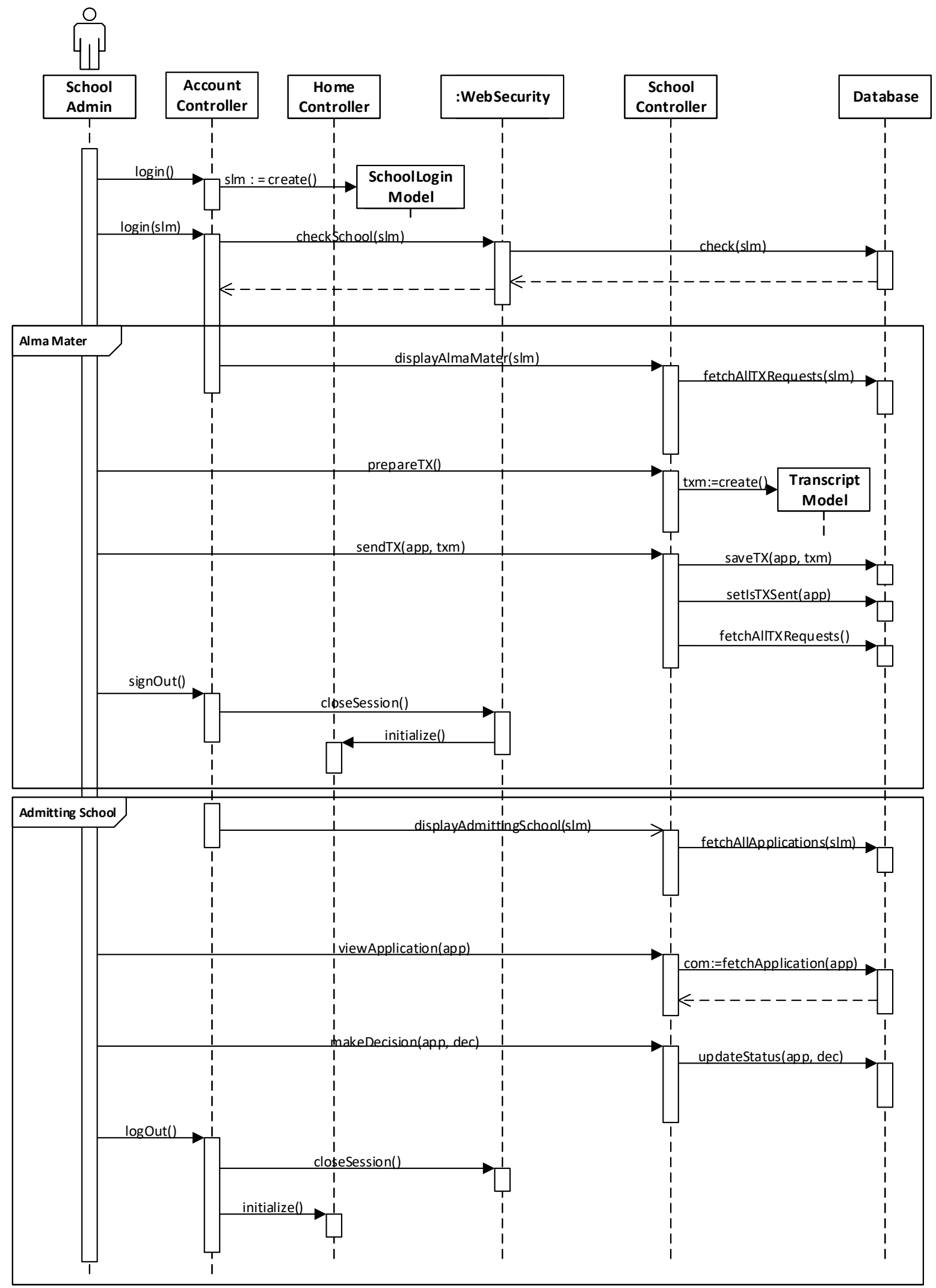

Figure 10: School Operations (UC4 and UC6) Realization 
'School' as previously explained could either be Alma Mater or Admitting School. Figure 10 shows the Alma Mater scenario first. In this scenario, an authenticated school is presented with a view and an access to all former students of the school who are currently applicants applying for postgraduate admissions into various schools. The other methods shown in the scenario are to prepare and effect the sending of a prepared transcript for a given applicant to the specified admitting school.

The second scenario shown in Figure 10 shows the methods required for an Admitting School to process an applicant's application and to make decision upon the application as necessary.

For both scenarios, (though not explicitly shown in the first), this framework incorporates an update on an applicant's dashboard. By this, it is necessary to indicate to an applicant when their requested transcript is sent by their alma maters and also to notify them of their admitting schools decision on their application.

\section{Provide Reference}

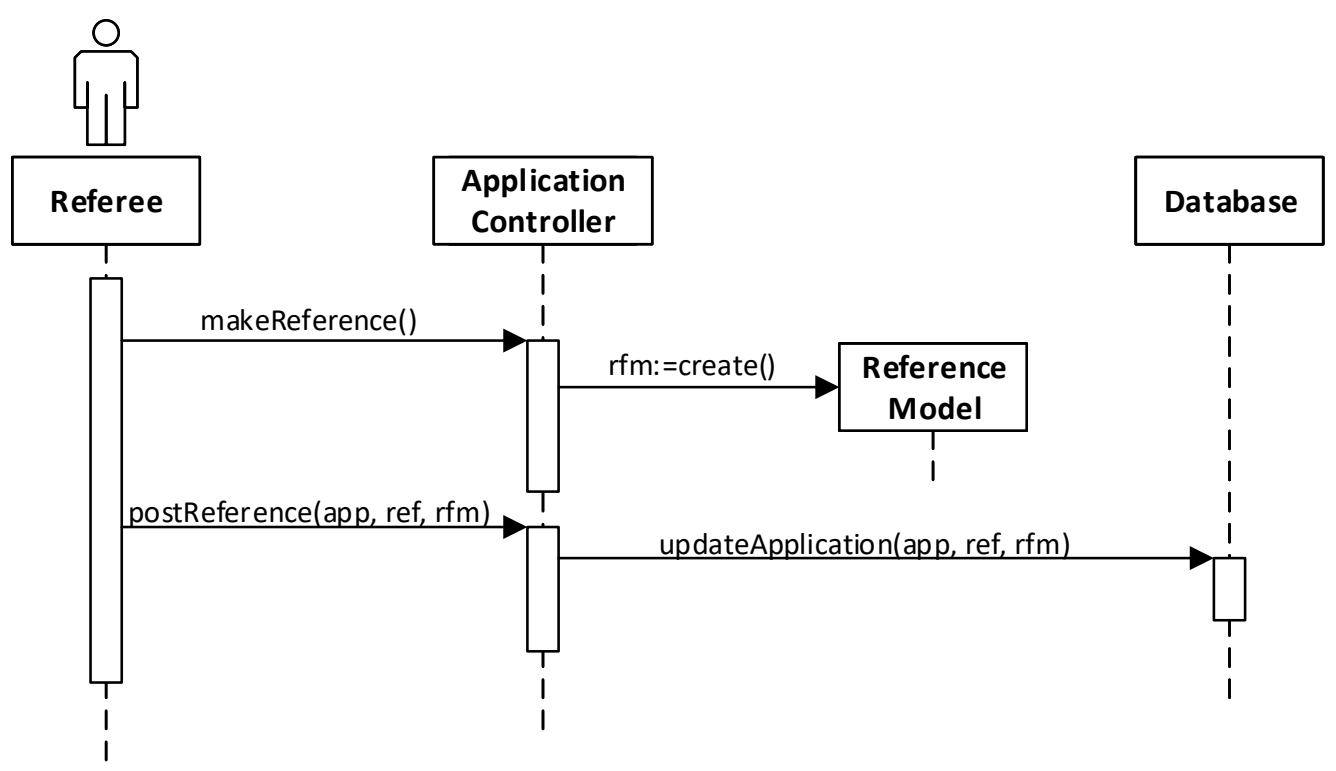

Figure 11: Provide Reference (UC5) Realization

Figure 11 shows the sequence diagram for the use case realization for Provide Reference. It simply shows two system event methods used to make reference and to post the made reference by the referee.

The arguments of the second method are quite important: one for identifying the applicant for whom the reference has been made, the second to identify the referee making the reference and the third to carry the reference itself. Although implementation approaches may differ based on developer choices and technologies, this framework considers these three arguments very important.

\section{SUMMARY, CONCLUSION AND RECOMMENDATION}

Summary: Web applications have become the primary source of information and transactions over the internet and this has led to high demand for continuously evolving web applications. Various technologies such as web forms have been used in development of web applications but the Model View Controller (MVC) software engineering architecture has been widely embraced. This is as an approach for developing Web-based systems that contain a server-side programming component, 
particularly for those requiring database access. MVC isolates the business logic from the user interface, with the goal of creating applications that are easier to manage and maintain because designers can modify the visual appearance of the application and programmers can modify the underlying business rules with fewer harmful side effects.

University administrators and stake holders depend on web applications for many of their operations including postgraduate admissions. Even though many institutions have independently developed systems to handle their postgraduate applications processing, this work sees a need to come up with a framework that can be used to efficiently carryout postgraduate admissions processing among all Nigerian institutions that will securely seamlessly integrate all necessary components such submission of applicants form, issuance and transfer of applicants transcripts, making and sending in of reference letters and admission request processing.

Perspectives from literature were considered. Schwinger and $\mathrm{Koch}^{9}$ note that it is not common to model Web applications in practice which they consider to be unfortunate. They argue that a model based approach provides a better alternative to the ad-hoc development of Web applications and its inherent problems including insufficient fulfillment of requirements, faulty specification, or missing system documentation. They posit that models represent a solid starting point for the implementation of a Web application taking into account static and dynamic aspects of the content.

Larman ${ }^{17}$ strongly advocates the learning core skills in object-oriented analysis and design (OOA/D) as the most essential component for the creation of well-designed, robust, and maintainable software. Beyond UML as a standard diagramming notation, he says there are more critical object-oriented things to learn; specifically, how to think in objects. The UML according to him is not OOA/D or a method, it is simply notation. He stated that it is not so helpful to learn syntactically correct UML diagramming and perhaps a UML CASE tool, but then not be able to create an excellent design, or evaluate and improve an existing one. This is the harder and more valuable skill.

Literature on student academic records and transcripts were covered including Student Information Management Systems and by extension the use of ICT in University Administrative system which reveals an increasing trend in the use of, and dependence on, automated computer systems for student records management among tertiary institutions. The Model View Controller architecture for software development was extensively discussed with the conclusion that the benefits of this architectural pattern far outweigh the initial rigors associated with it; separation of concerns in web development remains an undisputable need among developers today.

Of the various possible methodologies that exist for carrying out this work, particular choices were made. First, use case texts were written from which the system Use Case diagram was drawn. Domain class diagram for the system under study was drawn before proceeding to use sequence diagrams to achieve object design. Methods discovered from the sequence diagrams were collated into their respective classes and thus emerged the design class diagram (DCD).

Database design was done using T-SQL and LocalDB feature available in Microsoft Visual Studio 2012. Programming was done C\# language. The design was fully implemented using Internet Template with a number of tweaks. Design goals and objectives were thus realized. The implemented design was tested and the results were shown. The tests results were shown according to the system use cases including shots of relevant pictures. Exceptions and error handling are not included; the pictures are mainly for the MSS (main success scenarios). 


\section{CONCLUSION}

The use of ICT solutions among Nigerian universities may have progressed relatively far, but the need and the possibility to completely automate and integrate all the activities in the entire spectrum of postgraduate application and admission processes is shown in this study. The rigors of academically approaching software development for web applications may appear very hectic at first thought, but quality software should be developed only after sufficient ground work (by way of clearly drawn out frameworks) have been laid and properly documented. By this, developers could easily study the application and hence improve on certain areas if need be.

Contribution to Knowledge: The study has been able to provide a complete object oriented analysis and design (OOAD) for the domain of inter-university postgraduate admission and academic records processing beginning from the requirements to implementation. In as much as the framework may not be as robust as it can possibly be, fundamental system tools and behaviour for the system has been provided.

The Model-View-Controller architecture used for the system has been able to provide a clear view of the system, provided an easy way for other developers to understand it and hence made provision for implementing and extending the framework.

It is the firm belief of the researcher that given the depth of the analysis of the system and the design provided in the study, this framework cannot be ignored by experts and developers - at least it can serve as a reference work for alternative or complementing studies in this domain.

\section{RECOMMENDATION}

This framework can be further developed in almost every aspect of it. Of special note are the areas of:

i. Expanding the framework to include all other forms of postgraduate admissions apart from masters such as postgraduate diploma (PGD), masters in philosophy (M.Phil) and doctor of philosophy $(\mathrm{PhD})$.

ii. Incorporating interactive multimedia into the framework for purposes like prompting for every update/activity arising from the actors using the system.

iii. Funding and implementation of the framework by university system stakeholders (e.g. NUC) so as to nib at the bud most possibilities of errors and fraud associated with postgraduate application and admission systems in Nigeria.

\section{REFERENCES}

1. G, Saranya Devi, SMART - An Architecture Framework for Web Applications. A project for Master of Science Degree in the Graduate School of The Ohio State University, 2013.

2. N.Latifi, Architecture patterns in web applications and implementation of find local food. Final Year Project, Linnaeus University, School of Computer Science and Mathematics, 2010.

3. N. Heidke, J., Morrison \& J.Morrison, Assessing the Effectiveness of the Model View Controller Architecture for Creating Web Applications. Journal of Department of Computer Science University of Wisconsin-Eau Claire,2008 
4. R.E. Ubogu, Enhancing Entrepreneurship through Information Communication Technology (ICT). Book of Readings. Education and Training for Entrepreneurship. Delta State University, Abraka, 2011.

5. R.N.Osakwe, Problems and Prospects Of Using Information And Communication Technology For Record Keeping In Tertiary Institutions In Nigeria, Journal of Education and Practice www.iiste.org ISSN 2222-1735 (Paper) ISSN 2222-288X (Online),2012.3, 14.

6. University of Nigeria Nsukka., http://www.unn.edu.ng/guidelines-for-the-considerationof-application-for-inter-university-transfer/ 2015, (Accessed 11 $1^{\text {th }}$ March, 2016)

7. University of Ibadan, http://pgschool.ui.edu.ng /images/form/CURRENT $\% 20$ ADMISSION \%20GUIDE\%201516.pdf, 2015, (Accessed 11 ${ }^{\text {th }}$ March, 2016)

8. B.Y.Baha, Web Application Framework for Individuals at Risk of Type 2 Diabetes, 2013.

9. W.Schwinger \& N.Koch, Modeling web applications. Web Engineering,2006, 39-64

10. C.Larman, Applying UML and Patterns: An Introduction to Object Oriented Analysis and Design and Interative Development. Pearson Education India, 2012.

11. University of Cambridge - Academic References (2016) http://www.graduate.study .cam.ac.uk /how-do-i-apply/supporting-documentation/academic-references (Accessed $14^{\text {th }}$ March, 2016)

12. University of Cambridge- Transcripts (2016) http://www.graduate.study.cam.ac.uk/howdo-i-apply/supportingdocumentation/transcripts ,2016

13. S.R.Welch, Home Study, 1994.

14. ETX-NG. (2016). https://www.etx-ng.com/blog-challenges-with-requesting-and-issuingacademic-transcripts-how-we-help--14 (Accessed 11th March, 2016)

15. B.S.Clements, Building an Automated Student Record System: A Step-by-Step Guide for Local and State Education Agencies. Concordia University. (2016). https://www.concordia.ca/content/dam/concordia/offices/registrar/docs/Graduate_Applic ation_Form.pdf.

16. A.A.Eludire, The Design and Implementation of Student Academic Record Management System Research Journal of Applied Sciences, Engineering and Technology ISSN: 20407467, 2011,3(8) $707-712,2011$

17. S.Ngoma, An Exploration of the Effectiveness of SIS in Managing Student Performance. (A final year Dissertation in the School of Post Graduate Studies, Marlboro College, Vermont, USA) 2009.

18. S.Barrett, Information's Systems: An Exploration of Factors Influencing Effective Use. Journal of Research on Computing in Education, 1999. 
19. S.R.Bharamagoudar, R.B. Geeta \& S.G. Totad, Web based student information management system. International Journal of Advanced Research in Computer and Communication Engineering, 2013, 2(6), 2342-2348.

20. University Of Nairobi, http://www.uonbi.ac.ke /sites/default/ files/main _uon/Post\% 20Graduate\%20Application\%20form\%202012.pdf,2016.

21. University College London, https://www.ucl.ac.uk/prospective-students/graduate /apply/ application-form.pdf ,2016

22. F.Adam, 2012, Pro ASP.Net MVC 4

\section{* Corresponding Author: Dr.Oye N. D.}

Department of Computer Science MAUTECH- Yola Nigeria 